\title{
Saturn's auroral morphology and activity during quiet magnetospheric conditions
}

\author{
J.-C. Gérard, ${ }^{1}$ D. Grodent, ${ }^{1}$ S. W. H. Cowley, ${ }^{2}$ D. G. Mitchell, ${ }^{3}$ W. S. Kurth, ${ }^{4}$ J. T. Clarke, ${ }^{5}$ \\ E. J. Bunce, ${ }^{2}$ J. D. Nichols, ${ }^{2}$ M. K. Dougherty, ${ }^{6}$ F. J. Crary, ${ }^{7}$ and A. J. Coates ${ }^{8}$ \\ Received 10 July 2006; revised 30 August 2006; accepted 6 October 2006; published 16 December 2006.
}

[1] We report the results of a coordinated Hubble Space Telescope-Cassini campaign that took place between 26 October and 2 November 2005. During this period, Saturn's magnetosphere was in an expanded state and the solar wind was quiet, as indicated by the location of the magnetopause, in situ particle measurements, weak auroral SKR emission, and the generally low brightness of the aurora. We describe the morphology and dynamics of the aurora during this period in parallel with concurrent Cassini measurements. We show that the aurora exhibits considerable longitudinal structure and time variations over intervals of a few hours, in spite of the absence of observable external triggers and generally low intensity. In particular, enhancements of the dawn-morning oval are seen while no apparent indication of solar wind activity is observed. These features rotate at a speed corresponding to about $65 \%$ of the planet's angular velocity. We also describe energetic neutral atom measurements indicating that an ENA acceleration event occurred in the magnetotail on 26 October without any measured signature of solar wind activation. These observations suggest an intrinsically dynamical magnetosphere where injection of hot plasma occasionally takes place in the night or dawn sector during quiet magnetospheric conditions, possibly connected with either the Dungey or the Vasyliunas convection cycle.

Citation: Gérard, J.-C., et al. (2006), Saturn's auroral morphology and activity during quiet magnetospheric conditions, J. Geophys. Res., 111, A12210, doi:10.1029/2006JA011965.

\section{Introduction}

[2] The UV spectrometers on board Voyager 1 and 2 made the first observations of Saturn's aurora during the Saturn flybys. The HI Lyman- $\alpha$ line and $\mathrm{H}_{2}$ Lyman and Werner bands were observed in the polar regions of both hemispheres. The aurora appeared as a narrow ring located near $80^{\circ}$ latitude, with no measurable emission inside the oval [Broadfoot et al., 1981; Sandel and Broadfoot, 1981]. It showed temporal intensity variations (factors of $\sim 2-5$ ) [Sandel et al., 1982], suggesting a solar wind controlled aurora. Outbursts of Lyman- $\alpha$ were observed with the International Ultraviolet Explorer (IUE) spacecraft over a decade [Clarke et al., 1981; McGrath and Clarke, 1992],

\footnotetext{
${ }^{1}$ Laboratoire de Physique Atmosphérique et Planétaire, Université de Liège, Liège, Belgium.

${ }^{2}$ Department of Physics and Astronomy, University of Leicester, Leicester, UK.

${ }^{3}$ Applied Physics Laboratory, Johns Hopkins University, Baltimore, Maryland, USA.

${ }^{4}$ University of Iowa, Iowa City, Iowa, USA.

${ }^{5}$ Center for Space Physics, Boston University, Boston, Massachusetts, USA.

${ }^{6}$ Imperial College, London, UK.

${ }^{7}$ Southwest Research Institute, San Antonio, Texas, USA.

${ }^{8}$ Mullard Space Science Laboratory, University College London, London, UK.
}

Copyright 2006 by the American Geophysical Union. 0148-0227/06/2006JA011965\$09.00 also indicating a possible solar wind influence on Saturn's aurora. The Faint Object Camera on board the Hubble Space Telescope (HST) obtained the first image of the north aurora [Gérard et al., 1995]. Images collected with the Wide Field Planetary Camera (WFPC2) by Trauger et al. [1998] showed a northern auroral arc appearing mostly fixed in local time, generally brighter near the dawn limb but with variable brightness. Images of the south aurora obtained with the Space Telescope Imaging Spectrograph (STIS) [Gérard et al., 2004; Cowley et al., 2004; Clarke et al., 2005; Grodent et al., 2005] showed the presence of an auroral oval extending continuously from the midnight sector via dawn into the postnoon hours. The brightness of the main oval ranged from below the STIS threshold of $\sim 1 \mathrm{kR}$ of $\mathrm{H}_{2}$ emission up to $\sim 75 \mathrm{kR}$. The total electron precipitated power varied between 20 and 190 GW, comparable to the Earth's active aurora but about two orders of magnitude less than Jupiter. The dayside main oval was located between $70^{\circ}$ and $80^{\circ}$ latitude, generally but not always brighter and thinner in the morning than in the afternoon sector. The afternoon sector was characterized by more diffuse emission extending to higher latitudes. A spiral structure of the main oval was occasionally observed, as well as a bright spot fixed in the noon sector [Gérard et al., 2004], that was interpreted as the cusp signature of dayside reconnection [Bunce et al., 2005; Gérard et al., 2005]. Comparison of FUV auroral spectra with a synthetic model of electron-excited $\mathrm{H}_{2}$ was used to estimate the mean energy 
of the primary auroral electrons as $12 \pm 3 \mathrm{keV}$ [Gérard et al., 2004], based on a low-latitude model atmosphere relying on Voyager occultation measurements.

[3] As at other planets, the brightness, morphology, and dynamics of Saturn's aurora are controlled by complex processes that lead to plasma acceleration along the planetary magnetic field lines. Until recently, very few opportunities were available to coordinate solar wind measurements with auroral observations and to determine the magnetosphere's response to the solar wind. During January 2004, a coordinated campaign took place during which magnetic field, plasma, and radio wave instruments on board the Cassini spacecraft measured the in situ solar wind and embedded IMF and auroral radio emissions, while HST simultaneously observed the far ultraviolet aurora in Saturn's southern hemisphere [Clarke et al., 2005]. Observations showed a correlation between the brightness, the size of the main oval, and the solar wind characteristics. The shape of the auroral region, its level of corotation with the planet, and its brightness distribution were described by Clarke et al. [2005], Cowley et al. [2005], Bunce et al. [2005], and Grodent et al. [2005]. The relationship between the auroral FUV emission and kilometric radio (SKR) emission was analyzed by Kurth et al. [2005] who showed that both are correlated and that the SKR power also correlates with emission bandwidth. Observations covering nearly a complete Saturn rotation were collected during a low-field rarefaction interval, which followed a major compression during 1-5 January. The rarefaction region solar wind dynamic pressure was $\sim 0.003 \mathrm{nPa}$, while the IMF strength was typically $<0.1 \mathrm{nT}$ [Crary et al., 2005]. Concurrent images show a correspondingly highly expanded "quiet" auroral oval at $15^{\circ}-20^{\circ}$ colatitude, which contracted slowly over the following 8-day interval of low solar wind activity. Moderate brightenings were observed in the dawn and prenoon sector. A stronger enhancement and contraction of the oval was then observed after a modest compression of the magnetosphere by the solar wind (peak dynamic pressure $\sim 0.05 \mathrm{nPa}$ ), following which the oval reexpanded somewhat during a subsequent 8-day rarefaction region of intermediate characteristics (dynamic pressure $\sim 0.01 \mathrm{nPa}$ ), again with auroral brightenings at dawn and noon. A bright feature observed poleward of the main oval near noon during the latter interval was suggested by Gérard et al. [2006] to be due to lobe reconnection occurring under conditions of southward IMF, i.e., reconnection between southward pointing interplanetary magnetic field lines and open field lines of the magnetospheric tail lobes. Following this, a major 4-day solar wind compression event took place (peak dynamic pressures above $0.1 \mathrm{nPa}$ ), in which the auroral emission was considerably enhanced and expanded toward the pole in the dawn sector.

[4] Cowley et al. [2004] have suggested that Saturn's auroral oval is associated with a ring of upward directed field-aligned current flowing along the open-closed field line boundary generated by the difference in angular velocity between open magnetic field lines and outer magnetosphere closed field lines. This current is suggested to be carried by downward accelerated magnetospheric electrons which produce a bright discrete aurora at the boundary, possibly augmented by more diffuse emissions in the region immediately equatorward due to hot plasma production and precipitation in the Dungey and Vasyliunas cycles [Cowley et al., 2005]. Flow activity in these cycles will also produce greater flow shears and brighter aurora at the dawn boundary compared with dusk [Jackman and Cowley, 2006]. In this case, it is expected that the main oval aurora is strongly modulated by the upstream solar wind conditions. Using data from the January 2004 campaign, Badman et al. [2005] showed how the size of the oval and the variation of the open flux is controlled by the dynamical balance between dayside and nightside reconnection.

[5] The dynamical behavior of Saturn's magnetosphere was also demonstrated by Mitchell et al.'s [2005] measurements of abrupt increases in energetic atom flux originating from Saturn's magnetotail correlated with enhancements in Saturn kilometric radiation (SKR). The bursts of $\mathrm{H}^{+}$and $\mathrm{O}^{+}$ activity were interpreted as signatures of ion heating or acceleration somewhere between 20 and $30 R_{S}$. In one case the activity arose in isolation with no sustained magnetospheric activity preceding it but following a 17 -hour period of northward IMF. Another event involved repeated magnetotail activation triggered by the passage of an interplanetary shock and compression region. For these reasons, Mitchell et al. [2005] suggested that Earth-like substorms occur in Saturn's magnetotail with the fast rotating magnetic field adding phenomenology not seen at Earth.

[6] In this study, we report the results of a coordinated HST-Cassini campaign which took place between 26 October and 2 November 2005 during Cassini's Rev. 17. HST observations were made during quiet solar wind conditions, as indicated by the Cassini magnetic field and particle measurements and suggested by the generally low brightness of the aurora. We investigate the morphology and dynamics of the aurora during this period and show that the quiet state aurora exhibits considerable longitudinal structure and variations. We also describe energetic neutral atom measurements indicating that a substorm-like event occurred in the magnetotail, apparently in the absence of a significant solar wind pressure change. We conclude that Saturn has an intrinsically dynamical magnetosphere where injection of hot plasma can occasionally take place in the night or dawn sector even in the absence of external solar wind triggers.

\section{Auroral Images and Magnetospheric Context 2.1. HST Images}

[7] A total of 63 images of Saturn's southern auroras were obtained with the Advanced Camera for Surveys (ACS) in the period between 26 October and 2 November 2005. The images were organized in 9 HST orbits comprising seven contiguous exposures of $5.5 \mathrm{~min}$ each (see Table 1). During this campaign, the sub-Earth planetocentric latitude remained close to $-19.9^{\circ}$ and the subsolar latitude was $-17.6^{\circ}$. The average plate scale of about $0.032 \mathrm{arcsec} / \mathrm{pixel}$ provides a field of view of $35 \times 31 \operatorname{arcsec}^{2}$, which includes the full planetary disk and a fraction of the ring system. The point spread function (PSF) is approximately 2 pixels at half maximum, so the effective spatial resolution projected at Saturn is $\sim 400 \mathrm{~km}$. For a feature located at the central meridian longitude (CML) the smearing introduced by the planetary rotation is $3.1^{\circ}$ of longitude for each exposure. The light travel time to the Earth introduces a 
Table 1. Time and Characteristics of the Hubble Space Telescope ACS Exposures and Cassini Spacecraft Location

\begin{tabular}{cccccccc}
\hline & & & & & \multicolumn{3}{c}{$\begin{array}{c}\text { Cassini } \\
\text { Location }\end{array}$} \\
\cline { 6 - 7 } HST Orbit & Julian Day & Date, 2005 & Time, UT & CML, Start Time & $\mathrm{R}_{\mathrm{s}}$ & LT & UV Power, GW \\
\hline 1 & 299 & 26 Oct & $1603-1650$ & $285.8^{\circ}$ & 28.8 & 8.5 & 2.9 \\
2 & 300 & 27 Oct & $1250-1337$ & $267.9^{\circ}$ & 24.3 & 8.9 & 6.4 \\
3 & 300 & 27 Oct & $1738-1825$ & $70.1^{\circ}$ & 23.2 & 9.0 & 3.1 \\
4 & 301 & 28 Oct & $1249-1336$ & $358.2^{\circ}$ & 17.3 & 9.7 & 3.3 \\
5 & 301 & 28 Oct & $1737-1824$ & $160.4^{\circ}$ & 15.7 & 10.0 & 8.0 \\
6 & 302 & 29 Oct & $1112-1159$ & $34.4^{\circ}$ & 8.6 & 12.2 & 4.7 \\
7 & 302 & 29 Oct & $1600-1647$ & $196.6^{\circ}$ & 6.7 & 13.7 & 16.4 \\
8 & 306 & 2 Nov & $0620-0707$ & $233.3^{\circ}$ & 29.8 & 4.0 & 4.2 \\
9 & 306 & 2 Nov & $1108-1154$ & $35.5^{\circ}$ & 31.0 & 4.1 & 4.3 \\
\hline
\end{tabular}

76 min correction between the Cassini spacecraft time and the Earth time. The passband of the F125LP filter used for this campaign extends from 130 to $190 \mathrm{~nm}$ and responds to the $\mathrm{H}_{2}$ Lyman and Werner bands and mostly excludes the $\mathrm{H}$ I Lyman $-\alpha$ line. As part of the data reduction process, the images were flat-fielded using the most recent low-order flat field and pixel-to-pixel files provided by the Space Telescope Science Institute (STScI). Dark counts were subtracted using the appropriate STScI dark files. Following image processing, ACS counts were converted into flux units using a factor of $3.47 \times 10^{-3}$ count/s pixel for $1 \mathrm{kR}$ of $\mathrm{H}_{2}$ Lyman and Werner emission. This conversion factor is calculated based on a synthetic UV spectrum of $\mathrm{H}_{2}$ [Gérard et al., 2002]. The procedure used to determine the position of the center of the planet and the coordinates of each pixel was described by Gérard et al. [2004] and Grodent et al. [2005]. It is then possible to generate polar (orthographic) projections of the auroral emission. Maps are generated in a system fixed relative to the central meridian, simulating an observer looking from above the north pole through the planet into the southern hemisphere. The FUV power emitted in the region of visibility from Earth orbit was determined following careful subtraction of the sunlit disk background. For this purpose, a smooth disk image including auroral contribution was built from images showing weak aurora by interpolating the background intensity throughout the auroral region. The auroral counts are added and converted into power emitted in the $\mathrm{B}-\mathrm{X}$ and $\mathrm{C}-\mathrm{X}$ singlet transitions of $\mathrm{H}_{2}$.

\subsection{In Situ Cassini Measurements}

[8] The period of HST observations started on 26 October (day 299) when Cassini was moving inbound on Rev 17 at a distance of $\sim 29 \mathrm{R}_{\mathrm{S}}$ at 0830 local time. The orbit of the spacecraft during the observations is shown in Figure 1, projected onto Saturn's equatorial plane, in which it remained throughout the period. The times of the HST imaging intervals are shown by the crosses marked on the spacecraft track. During this campaign, the magnetic field was continuously measured by the MAG instrument on board Cassini [Dougherty et al., 2004], as shown in the bottom four panels of Figure 2. These show the components of the field in spherical polar coordinates referenced to the planet's spin axis (positive northward), together with the magnitude of the field, over the interval from day 297 to
308 inclusive (24 October to 4 November). The "Cassini" model of Saturn's internal field derived by Dougherty et al. [2005] has been subtracted from the component data to reveal the fields due to "external" currents alone (e.g., the magnetopause, tail, and ring current fields), while the total measured field strength is shown in the bottom panel, together with the "Cassini" model internal field strength (green dashed line) for purposes of comparison. The top two

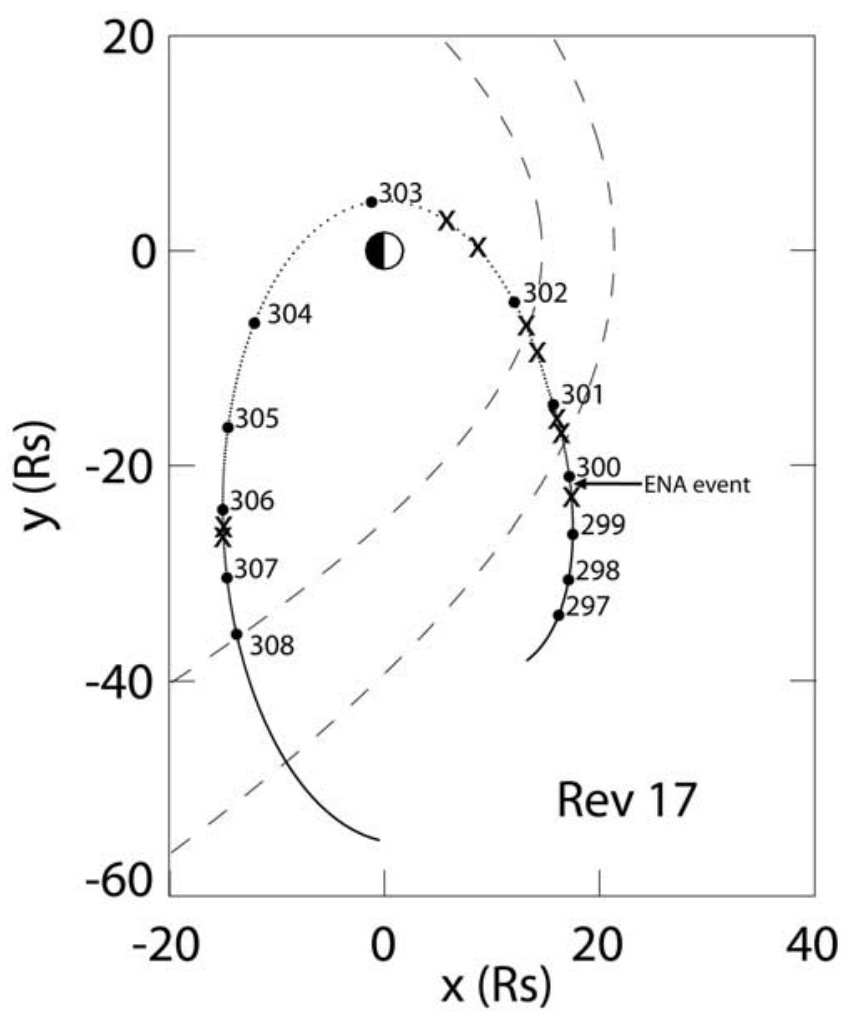

Figure 1. Segment of Cassini's orbit in the X-Y plane (Saturn's equatorial plane) for part of revolution 17. The Sun is located to the right of the plot. The dashed lines represent the expected location of the magnetopause for low and high solar wind pressure based on the Slavin et al. [1985] model. The crosses indicate the position of Cassini when Hubble Space Telescope (HST) images were taken and the arrow shows its location at the time when the energetic neutral atom (ENA) storm was detected. 


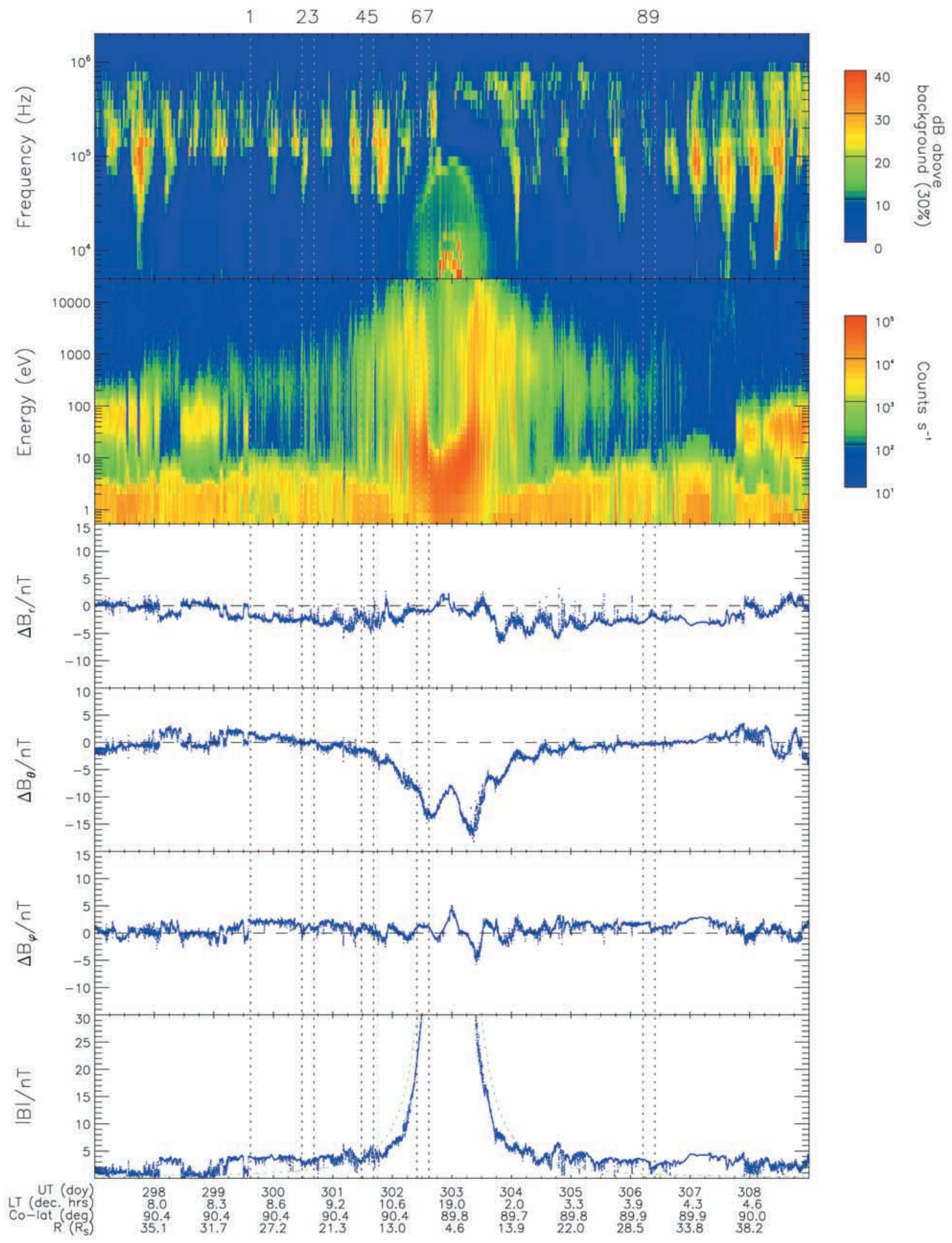

Figure 2 
panels show color spectrograms of the radio wave power in the frequency band from $5 \mathrm{kHz}$ to $\sim 2 \mathrm{MHz}$ measured by the RPWS instrument [Gurnett et al., 2004] and the electron count rate in the energy range from $\sim 0.5 \mathrm{eV}$ to $\sim 30 \mathrm{keV}$ measured by CAPS/ELS [Young et al., 2004]. Position data at the foot of the figure indicate the spacecraft's radial distance, local time, and colatitude from the northern spin axis. The corresponding start times of the HST observation intervals are indicated in the figure by the vertical dashed lines marked with the orbit number at the top of the plot, where account has been taken of the 76 min Saturn-Earth propagation time.

[9] At the beginning of the period shown in Figure 2, starting day 297, Cassini was inbound in the morning sector (LT $=7.6$ hours) as indicated in Figure 1. The spacecraft at that time was located in the magnetosheath, though there were two $\sim 1$ hour excursions across the bow shock into the solar wind on that day. The IMF strength in the solar wind was $\sim 0.1-0.3 \mathrm{nT}$, indicative of rarefaction region conditions, comparable with the "intermediate" rarefaction observed during 19-25 January 2004 in the January 2004 HST campaign, and greater than in the initial January 2004 rarefaction where field strength decreased below $0.1 \mathrm{nT}$. The corresponding field strengths in the magnetosheath were $\sim 0.5-2 \mathrm{nT}$. Initial entry into the magnetosphere took place near the beginning of day 298 at a radial distance of $\sim 34.8 \mathrm{R}_{\mathrm{S}}$ (see orbit plot). In total, seven main magnetopause transitions occurred inbound, the last at $\sim 1400$ UT on day 299 at a radial distance of $29.2 \mathrm{R}_{\mathrm{S}}$. If we interpret these boundaries as being in approximate pressure balance with the prevailing solar wind, then the dynamic pressure inferred using the Arridge et al. [2006] model varies between $\sim 0.01 \mathrm{nPa}$ for the furthest encounter and $\sim 0.02 \mathrm{nPa}$ for the nearest. The corresponding subsolar magnetopause positions are $\sim 28$ and $\sim 24 \mathrm{R}_{\mathrm{S}}$. These dynamic pressure values are again comparable with the "intermediate" rarefaction region in January 2004 and greater than the initial rarefaction region observed during that campaign.

[10] No signature of any major magnetospheric dynamic activity is observed from the in situ data on the inbound pass. In particular, there is no evidence of field or plasma changes that might be indicative of major solar wind events (e.g., compressions). We thus infer that during HST orbits 1-5 the magnetosphere was relatively quiescent and expanded under intermediate rarefaction conditions. In common with other such Cassini passes, the main in situ feature is the occurrence of oscillations in the field and plasma near the planetary period, together with related regular oscillations in the SKR power (to be discussed below). The field oscillations are primarily in the radial and azimuthal com- ponents, with lesser (but not zero) effects in the colatitudinal component. These were first noticed in Pioneer and Voyager data by Espinosa and Dougherty [2000] and Espinosa et al. [2003] and are found to be ubiquitous in Cassini data [Cowley et al., 2006]. These oscillations are believed to be associated with a corotating anomaly at the planet (not directly identified to date) the perturbations due to which propagate outward into the magnetosphere to form a corotating spiral pattern.

[11] Moving toward periapsis at $\sim 4.6 \mathrm{R}_{\mathrm{S}}$ at the end of day 302, the principal magnetic feature is the usual growth of a large negative $\Delta B_{\theta}$ field produced by the ring current, peaking in the inner region at about -17 nT. HST orbits 6 and 7 occurred when Cassini was located at distances of $\sim 7-9 \mathrm{R}_{\mathrm{S}}$ near the noon meridian, near the inner edge of the ring current where the $\theta$ field perturbation is a maximum. The ring current inner edge is typically located at such distances [Connerney et al., 1983; Bunce and Cowley, 2003]. At this time the spacecraft was thus located deep within the quasi-dipolar magnetosphere such that we would not expect to observe the effect of dynamics associated with the solar wind, outer magnetosphere, and tail that might connect with the main oval auroras.

[12] Oscillatory field and plasma behavior were also observed throughout the outbound pass, encompassing the interval on day 306 when the last HST images were collected. A rather unusual increase in the dawn flank magnetic field strength was observed at the end of days 306 and during day 307 when Cassini appears to exit from the plasma sheet into the lobe and then to re-enter the plasma sheet. The latter period also coincided with a hotter electron plasma, as observed in the CAPS/ELS data. These observations are indicative of a probable compression by the solar wind. The outbound magnetopause crossing then occurred at the end of day 307, at a radial distance of $\sim 38 \mathrm{R}_{\mathrm{S}}$ and a local time of 4.6 hours. This position indicates a solar wind dynamic pressure of $\sim 0.08 \mathrm{nPa}$ according to Arridge et al. [2006] model, consistent with a significant increase in dynamic pressure between the inbound and the outbound passes.

[13] The spectral intensity of the SKR emission over the same time period is shown at the top of Figure 2. The emission is regularly pulsed at the planetary period with occasional minor disturbances possibly linked to magnetospheric activity. Examples of pattern disruption are seen on days 297, 304, 307, and 308, but none is coincident with any of the HST exposures. Globally, the SKR data show the periodic rotationally driven pulsation dominates and do not provide evidence of significant auroral acceleration activity. We thus conclude on the basis of both the SKR and the in

Figure 2. Plot of Cassini measurements made in the interval from days 297 to 308 of 2005. The top panel shows a color spectrogram of the radio wave power in the frequency band from $5 \mathrm{kHz}$ to $\sim 2 \mathrm{MHz}$, while the second panel similarly shows the electron flux in the energy range from $\sim 0.5 \mathrm{eV}$ to $\sim 30 \mathrm{keV}$. The bottom four panels show the magnetic field components in spherical polar coordinates referenced to the planet's spin axis (positive north), together with the total field strength. The "Cassini" model of Saturn's internal field has been subtracted from the field component data, while the bottom panel shows the total measured field strength together with that of the "Cassini" internal field model (green dashed line). Position data at the foot of the figure indicate the spacecraft's radial distance in Saturn radii ( $\left.\mathrm{R}_{\mathrm{S}}\right)$, the local time (decimal hours), and colatitude measured from the northern spin axis (degrees). The start times of the HST observation intervals are indicated in the figure by the vertical dashed lines marked with orbit numbers at the top of the plot, where account has been taken of the 76 min Saturn-Earth propagation time. 


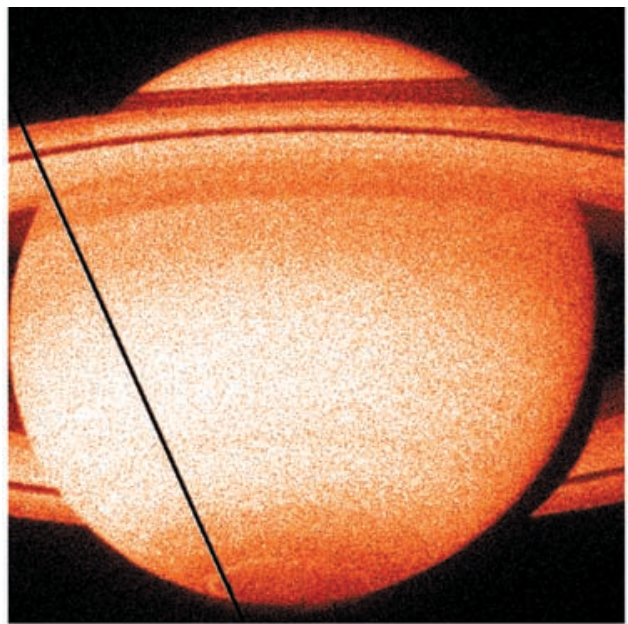

Oct. 26, 2005

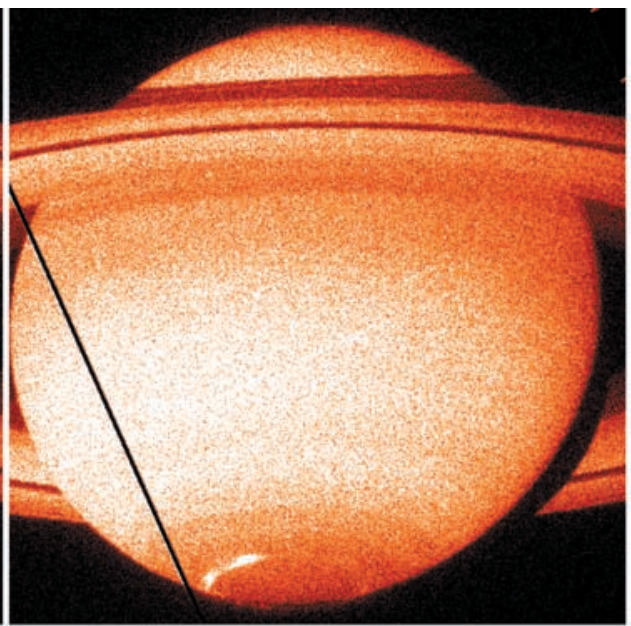

Oct. 29,2005

Figure 3. Examples of images of Saturn's aurora obtained during HST orbit 1 (26 October) and 7 (28 October). The dark line is the trace of an ACS occulting bar.

situ field and plasma data that this campaign took place during a quiescent solar wind period with only low-level activity.

\section{Observations}

[14] We first analyze the auroral morphology and dynamics observed in the FUV during this period. We then describe energetic neutral atom measurements made by Cassini and show evidence for an ion acceleration episode observed on 26 October, approximately 5 hours after the first HST exposures were taken.

\subsection{Auroral Morphology and Dynamics}

[15] All 63 exposures were obtained with the same ACS filter, making comparison of the morphology and relative brightness straightforward. Two examples of HST images are shown in Figure 3. The first one is the first exposure collected during HST orbit 1 (day 299). It shows a thin continuous discrete oval standing out against the sunlit disk and extending from the dawn to the dusk limb. The second example shows the first image of HST orbit 7 (day 302) where the dawn and morning oval is strongly enhanced in comparison with the weaker afternoon sector.

[16] Figure 4 presents polar projections for the first, fourth, and seventh image of selected HST orbits. On each plot, local noon is directed toward the bottom, midnight at the top, dawn to the left and dusk to the right. Figure $4 \mathrm{a}$ (orbit 1) shows a weak oval in the morning located close to $75^{\circ}$. Parallels are distant by $10^{\circ}$ and the meridians are separated by $10^{\circ}$ of longitude. In the afternoon sector, the emission is located at higher latitude with a $2^{\circ}-3^{\circ}$ discontinuity around noon. On this image as in the other ones obtained during this campaign, no enhanced noon emission is observed. We note that the sensitivity of the ACS camera is comparable to STIS which was used by Gérard et al. [2005], Clarke et al. [2005], and Grodent et al. [2005], who observed occasionally enhanced cusp emission. The presence of cusp emission has been interpreted [Gérard et al., 2004, 2005; Bunce et al., 2005] as a signature of dayside reconnection at low latitudes or in the lobes. Three images obtained 21 hours later during HST orbit 2 are shown in Figure $4 \mathrm{~b}$. The overall auroral morphology was similar, but a pronounced enhancement was occurring in the morning sector. The weak oval extending from dawn to dusk is quite similar in location and brightness to that of orbit 1 . However, a bright feature extending from 0800 to $1000 \mathrm{LT}$ clearly stands up above the rest of the oval. This enhanced emission shows two intensity peaks separated by $\sim 15^{\circ}$ of longitude. Over the $\sim 50 \mathrm{~min}$ of observations, the spots drift eastward toward the central meridian at a rate of about $65 \%$ of the planetary spin rate, indicating that these are not cusp enhancements on the oval. We note that this corotation lag is similar to earlier determinations based on comparison of images on successive HST orbits, that is determined over time spans of about $90 \mathrm{~min}$ [Clarke et al., 2005; Grodent et al., 2005]. During this motion, the emission rate decreases and the leading edge moves poleward by $\sim 2^{\circ}$. Such subcorotating blobs have been previously interpreted as due to plasma injections initially on the nightside, due to field line reconnection associated with the Dungey or the Vasyliunas cycles [Cowley et al., 2005]. Five hours later (orbit 3), the oval (not shown) was hardly detectable and the associated power reached the lowest value of all existing STIS or ACS observations. Auroral emission measured on orbit 4 is quite weak but nearly homogeneous in longitude. Note that the apparent extension of the nightside emission toward low latitudes is an effect of the polar projection of emission located close to the dawn and dusk limbs. In the three polar projections of orbit 5 shown in Figure 4d the oval is clearly brighter in the prenoon than in the afternoon sector. Weak but clearly present emission is observed in the evening sector at latitudes of about $75^{\circ}-82^{\circ}$. This feature is possibly associated with the inner segment of the spiral structure described before, as in the work of Gérard et al. [2004, Figure 3] but with a poorer visibility of the night sector resulting from the smaller tilt angle of Saturn than in 2001. The two orbits (6 and 7) of 29 October show a dramatic change over the $\sim 5$ hours separating the two sets of exposures. A thin and weak oval appears in the morning 

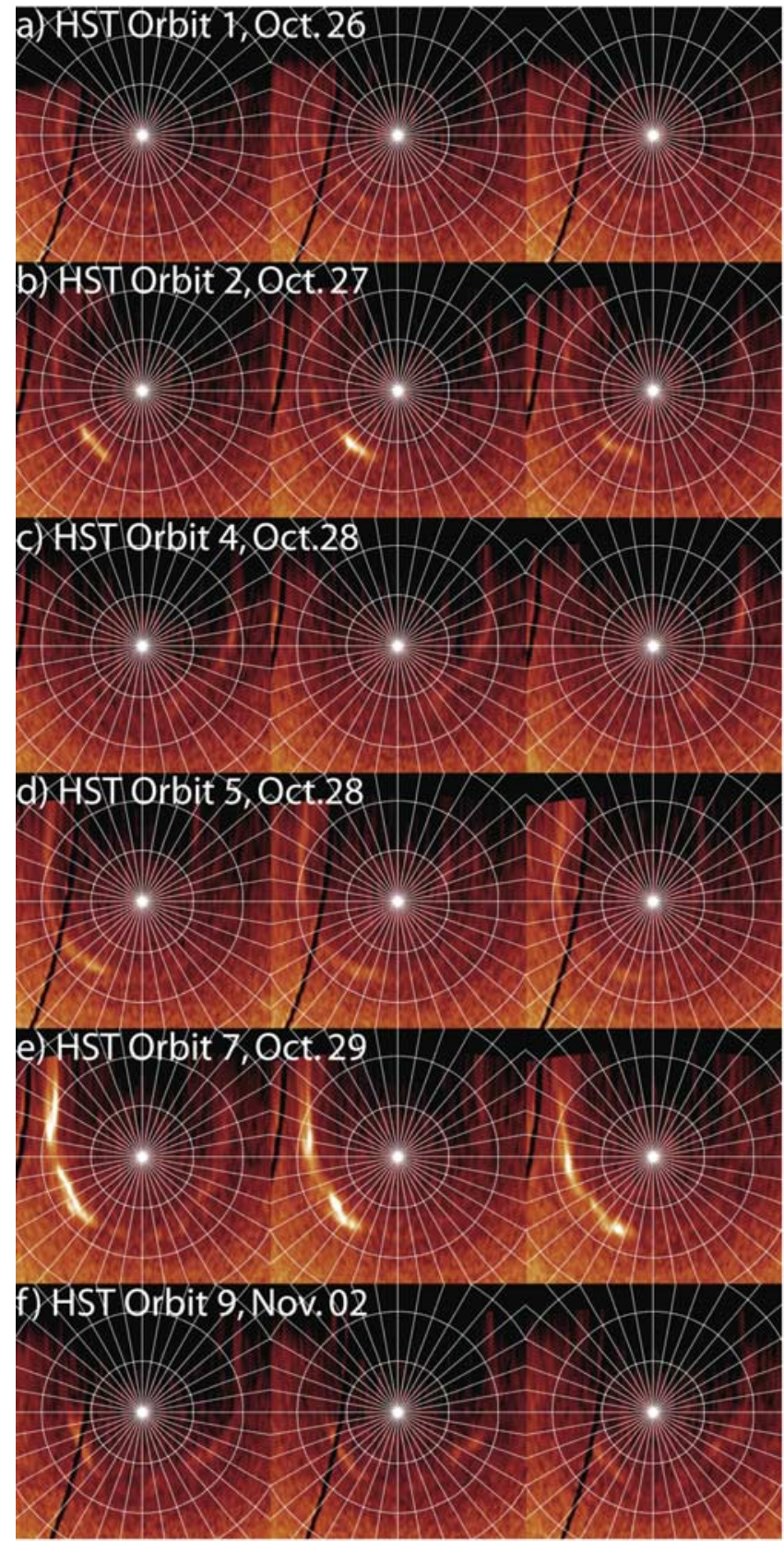

Figure 4. Selection of polar (orthographic) projections of HST-ACS images of Saturn's south FUV aurora obtained during the campaign. The first, fourth, and seventh images taken on HST orbits $1,2,4,5,7$, and 9 are shown. The images have been projected on a polar view from the pole to $30^{\circ}$ colatitude where the south is seen by a virtual observer above the north pole, looking through the planet. In these viewing conditions, the south pole is at the center, the direction of the Sun (1200 LT) is oriented toward the bottom, dawn (0600 LT) to the left, and dusk (1800 LT) to the right. The parallels are separated by $10^{\circ}$ of latitude and the meridians by $10^{\circ}$ of longitude. The same color scale is used for all projections. The dark line is the trace of an ACS occulting bar. and afternoon sectors during orbit 6 . The morning emission is considerably broader and brighter during orbit 7 and characterized by two bright spots whose shape and relative intensity vary over the 7 consecutive exposures. The associated FUV power of $\sim 16 \mathrm{GW}$ originates predominantly from the prenoon sector. Projections for orbit 7 show that the intense morning precipitation extends over more than $80^{\circ}$ of longitude and is longitudinally structured with two regions of enhanced auroral emission. The second and third projections show a marked wavy structure. A time evolution is observed in the longitudinal brightness distribution. The leading edge of the precipitation region moves toward the central meridian at a rate of $\sim 70 \%$ of the planet's motion. Images on orbit 8 of 2 November show the spiral morphology that was first described by Gérard et al. [2004], in which with increasing local time the aurora winds from highest latitudes in the dusk sector to lower latitudes at dawn and noon. Cowley et al. [2005] explained these features as resulting from the combined action of magnetic field line reconnection and rotation. Auroral spirals are formed by hot plasma precipitation on newly-closed flux tubes, and expected to occur when the tail reconnection rate exceeds the dayside rate. Finally, the last set of exposures (orbit 9 shown in Figure 4f) shows weak auroral emission with longitudinal intensity variations along the oval. By that time, Cassini was moving outbound at a distance of $\sim 31$ Rs at $\sim 0400$ LT

[17] The total count rate above background may be directly converted into units of total emitted power in the ACS passband. Using a measured spectrum of Saturn's aurora, the total $\mathrm{H}_{2}$ emission in the Lyman and Werner bands may be calculated using a conversion factor of $10 \mathrm{kR} /$ incident $\mathrm{mW} / \mathrm{m}^{2}$ of electron precipitation [Gérard and Singh, 1982; Waite et al., 1983; Grodent et al., 2001]. Figure 5 shows the time evolution of the radiated power in the $\mathrm{H}_{2}$ bands associated with the average of the seven images of each HST orbit, also listed in Table 1. The radiated power ranges between 3 and $16 \mathrm{GW}$, corresponding to a precipitated power of about 2 to $11 \times 10^{10} \mathrm{~W}$. These radiated power values are comparable to the 1.5-26 GW of UV radiation observed with STIS between 1997 and 2001.The smaller planetary tilt angle of these observations does not offer the same visibility of the nightside oval, which may lead to a relative underestimate of the apparent radiated power in comparison to earlier observations. The present powers are comparable with the 4-20 GW of UV emission observed during solar wind rarefaction conditions [Clarke et al., 2005; Badman et al., 2005], as may be expected from the low quiet solar wind conditions prevailing during this campaign. We note however that the disk background count is relatively large in comparison with the contribution of the weak aurora, which makes quantitative comparison between the two cameras somewhat uncertain.

\subsection{Plasma Acceleration Event in the Magnetotail}

[18] We now describe the characteristics of a plasma acceleration event that was observed during the time period spanned by the HST observations and examine possible relationships with the observed auroral activity. Energetic neutral atom measurements were made by the Ion and Neutral Camera (INCA) sensor on Cassini during this campaign. None of the ENA observations during this period 


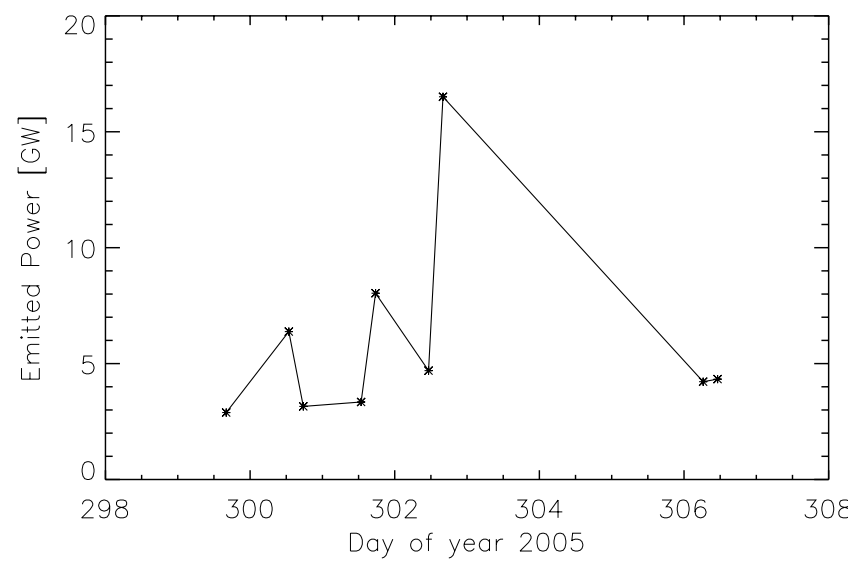

Figure 5. Time evolution of the emitted FUV auroral power determined from the average of the exposures taken on each HST orbit.

was out of the ordinary, with the exception of one event. An increase of the ENA flux from the magnetotail was observed on day 299 (26 October) starting at 2200 UT and extending into early day 300. Figure 6 shows an increase in the oxygen flux in all energy channels between 32 and $200 \mathrm{keV}$ but more pronounced in the $96-144 \mathrm{keV}$ channel where the flux rose by more than one order of magnitude. A similar enhancement was also observed in the $H$ flux. Figure 7 shows a sequence of frames illustrating the time evolution of the $20-50 \mathrm{keV} \mathrm{H}$ atom flux between 2100 and
2300 UT. During this period, the Cassini vantage point was near $0800 \mathrm{LT}$ at a distance of $27 \mathrm{R}_{\mathrm{S}}$. Figure 7 indicates that the spacecraft was then inside the inflated magnetosphere. The ENA count rate before 2200 UT is typical of conditions usually observed in the absence of an event. Both the $\mathrm{H}$ and $\mathrm{O}$ fluxes are coming from the vicinity of the equatorial plane at a distance of less than $20 \mathrm{R}_{\mathrm{S}}$. At $2200 \mathrm{UT}$ a sudden brightening is observed in the direction of the magnetotail, which indicates a sudden acceleration of the plasma ions along a line of sight that includes the magnetotail close to midnight local time at a radial distance near $20 \mathrm{R}_{\mathrm{S}}$. Following the peak in brightness, the hot plasma population accelerated in the event appears to be picked up in the corotation flow around the planet, sweeping from the night side through the morning, past the spacecraft meridian, and into the dayside. After this, it disappears and can no longer be seen. The disappearance of this event as it moved toward the dayside is not caused by a lack of neutrals. Two possibilities may be considered: (1) the injected plasma simply "cooled" below the energies of the ENA instrument. That is, the acceleration event was short-lived, and the hot ion population expanded, drifted, and dispersed until it is no longer visible above background; or (2) it went out through the magnetopause. This might be caused by reconnection, but it could also disappear through cross-field drifts across the magnetopause, or the flux tubes might get caught up in boundary layer flow and convected downtail. The disappearance as they reach the dayside is not uncommon among other such ENA events. Sometimes, as described by Mitchell et al. [2005], they get around to the dusk flank

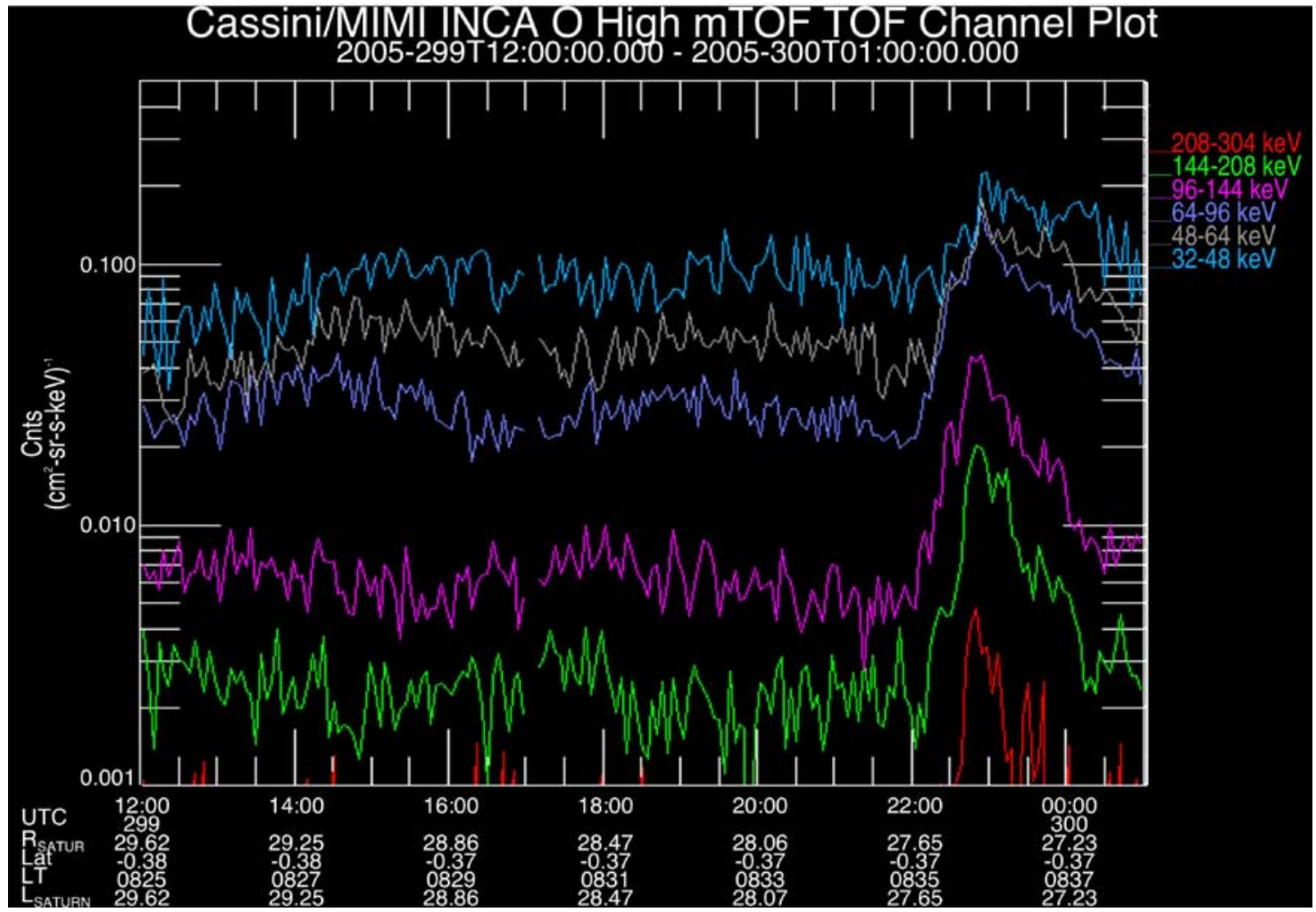

Figure 6. Variation of the flux of energetic O atoms measured by the INCA instrument during day 299 (26 October) 2005 in different energy ranges. The abrupt increase observed near 2200 UT in all energy channels is presumably caused by an acceleration event in the magnetotail at about $20 \mathrm{R}_{\mathrm{S}}$. 


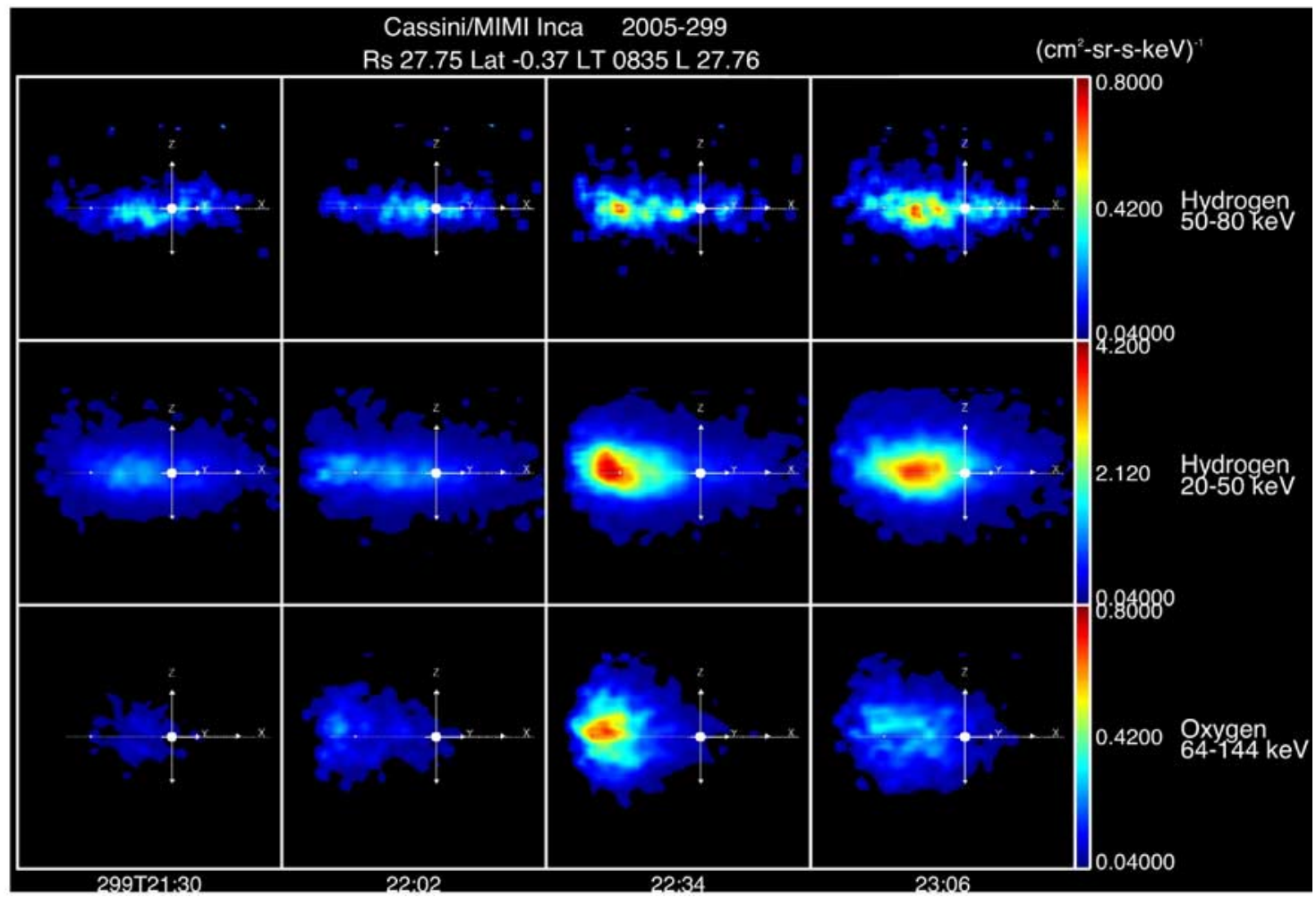

Figure 7. Time sequence of the spatial distribution of the ion acceleration event as seen from Cassini by the INCA instrument on day 299 between 2130 and 2306 UT. From top to bottom: H 50-80 keV, H 20$50 \mathrm{keV}, \mathrm{O} 64-144 \mathrm{keV}$. The axis origin is at the position of Saturn and the vertical arrow indicates the direction of the normal to the Cassini orbital plane. The ENA count rate (in $\mathrm{cm}^{-2} \mathrm{~s}^{-1} \mathrm{sr}^{-1} \mathrm{keV}^{-1}$ ) is color-coded on a linear scale. The ENA event is observed to follow a low activity period.

before completely disappearing but frequently fade out after a few hours. Consequently, the auroral signature associated with the hot plasma cloud would be expected to be considerably diminished at the time of the following HST exposure, almost 13 hours after the center time of the ENA burst.

[19] The phenomenology of the event described here is quite similar to the isolated "substorm event" described by Mitchell et al. [2005] with no preceding magnetospheric activity or triggering solar wind event. However in the present case, unlike the other two cases discussed by Mitchell et al., no enhancement of kilometric radiation was observed during the burst of ion activity. The event possibly did not remain active by the time it reached the L-shell where SKR is generated. A general increase is observed in the SKR intensity at the time of the event, which is in phase with the rotation modulated SKR that characterizes the whole period. It is possible that the rotational modulation of SKR is stronger than that created by the substorm which thus masked by the diurnal SKR pattern. The Cassini magnetometer did not record any evidence of a magnetic field strength increase at the time of ENA in response to this remotely observed plasma acceleration event in the magnetotail.

\section{Summary and Conclusions}

[20] Magnetometer, energetic particle, and SKR measurements indicate that Saturn's magnetosphere was expanded and fairly quiescent during this campaign. In spite of the apparent absence of any solar wind triggers, and the generally undisturbed appearance of the auroral oval, some of the HST images clearly show the presence of spirals and subcorotating auroral patches. These have been suggested to be due to impulsive injections of hot plasma from the magnetospheric tail in the nightside sector associated with the Dungey or the Vasyliunas cycles. They are followed by subcorotation into the dayside sector in the outer magnetosphere due to magnetosphere-ionosphere momentum coupling [Cowley et al., 2005; Grodent et al., 2005]. Estimates indicate that this hot plasma generally forms a layer a few Saturn radii wide just inside the dayside magnetopause. During the early phase of the campaign, an ion heating or acceleration event in the magnetotail produced an $\mathrm{H}^{+}$and $\mathrm{O}^{+}$enhancement over a few hours. No auroral signature was observed in the HST images. As mentioned before the auroral signature associated with the cloud is expected to have strongly diminished by the time of the next HST image. In any case, assuming that the local time of the ENA burst was close to $0230\left(90^{\circ}\right.$ to the left of the line of sight), drifting at $65 \%$ of corotation, the burst of plasma would have reached a local time close to midnight by the time of the next Hubble image. Near midnight, its auroral signature, if it had one, would be out of HST visibility.

[21] Observations of Saturn's southern FUV aurora were made with the Hubble Space Telescope in parallel with in situ measurements of the solar wind plasma characteristics and embedded interplanetary magnetic field during quiet magnetosphere conditions. In terms of existing theories, the 
observed auroral morphology implies the continual but intermittent occurrence of dynamic phenomena associated with either the solar wind or with rotation through the Dungey and/or Vasyliunas cycles during a relatively quiescent interval. The activity results in overall modulations of the auroral precipitation intensity around the oval, variable dawn-dusk asymmetries in brightness, and subcorotating patches and arcs such as observed during this campaign.

[22] Acknowledgments. This work is based on observations with the NASA/ESA Hubble Space Telescope, obtained at the Space Telescope Science Institute (STScI), which is operated by the AURA, Inc. for NASA. JCG and DG acknowledge support from the Belgian Fund for Scientific Research (FNRS). The PRODEX program of ESA provided financial support for this research to the University of Liège. SWHC was supported by PPARC grant PPA/G/O/2003/00013. The research at The University of Iowa was supported by NASA through contract 1279973 with the Jet Propulsion Laboratory and the work at MSSL-UCL was funded by PPARC. This research was also supported by grant GO-10506.01-A from the Space Telescope Science Institute and from NASA to Boston University.

[23] Wolfgang Baumjohann thanks W. R. Pryor and another reviewer for their assistance in evaluating this paper.

\section{References}

Arridge, C. S., N. Achilleos, M. K. Dougherty, K. K. Khurana, and C. T. Russell (2006), Modeling the size and shape of Saturn's magnetopause with variable dynamic pressure, J. Geophys. Res., 111, A11227, doi:10.1029/2005JA011574

Badman, S. V., E. J. Bunce, J. T. Clarke, S. W. H. Cowley, J.-C. Gérard, D. Grodent, and S. E. Milan (2005), Open flux estimates in Saturn's magnetosphere during the January 2004 Cassini-HST campaign, and implications for reconnection rates, J. Geophys. Res., 110, A11216, doi:10.1029/2005JA011240.

Broadfoot, A. L., et al. (1981), Extreme ultraviolet observations from Voyager 1 encounter with Saturn, Science, 212, 206

Bunce, E. J., and S. W. H. Cowley (2003), A note on the ring current in Saturn's magnetosphere: Comparison of magnetic data obtained during the Pioneer-11 and Voyager-1 and -2 fly-bys, Ann. Geophys., 21, 661 .

Bunce, E. J., S. W. H. Cowley, and S. E. Milan (2005), Interplanetary magnetic field control of Saturn 's polar cusp aurora, Ann. Geophys. 23,1405 .

Clarke, J. T., H. W. Moos, S. K. Atreya, and A. L. Lane (1981), IUE detection of bursts of H Ly $\alpha$ emission from Saturn, Nature, 290, 226.

Clarke, J. T., et al. (2005), Morphological differences between Saturn's ultraviolet aurorae and those of Earth and Saturn, Nature, 433, 717.

Connerney, J. E. P., M. H. Acuña, and N. F. Ness (1983), Currents in Saturn's magnetosphere, J. Geophys. Res., 88, 8779

Cowley, S. W. H., D. M. Wright, E. J. Bunce, A. C. Carter, M. K. Dougherty, G. Giampieri, J. D. Nichols, and T. R. Robinson (2006), Cassini observations of planetary-period magnetic field oscillations in Saturn's magnetosphere: Doppler shifts and phase motion, Geophys. Res. Lett., 33, L07104, doi:10.1029/2005GL025522.

Cowley, S. W. H., E. J. Bunce, and R. Prangé (2004), Saturn's polar ionospheric flows and their relation to the main auroral oval, Ann. Geophys., 22, 1379 .

Cowley, S. W. H., S. V. Badman, E. J. Bunce, J. T. Clarke, J.-C. Gérard, D. Grodent, C. M. Jackman, S. E. Milan, and T. K. Yeoman (2005), Reconnection in a rotation-dominated magnetosphere and its relation to Saturn's auroral dynamics, J. Geophys. Res., 110, A02201, doi:10.1029/ 2004JA010796.

Crary, F. J., et al. (2005), Solar wind dynamic pressure and electric field as the main factors controlling Saturn's aurorae, Nature, 433, 720.

Dougherty, M. K., et al. (2004), The Cassini magnetic field investigation, Space Sci. Rev., 114, 331

Dougherty, M. K., et al. (2005), Cassini magnetometer observations during Saturn orbit insertion, Science, 307, 1266.

Espinosa, S. A., and M. K. Dougherty (2000), Periodic perturbations in Saturn's magnetic field, Geophys. Res. Lett., 27, 2785.
Espinosa, S. A., D. J. Southwood, and M. K. Dougherty (2003), Reanalysis of Saturn's magnetospheric field data view of spin-periodic perturbations, J. Geophys. Res., 108(A2), 1085, doi:10.1029/2001JA005083.

Gérard, J.-C., and V. Singh (1982), A model of energetic electrons and EUV emission in the Jovian and Saturnian atmospheres and implications, J. Geophys. Res., 87, 4525.

Gérard, J.-C., V. Dols, D. Grodent, J. H. Waite, G. R. Gladstone, and R. Prangé (1995), Simultaneous observations of the Saturnian aurora and polar haze with the HST/FOC, Geophys. Res. Lett., 22, 2685.

Gérard, J.-C., J. Gustin, D. Grodent, P. Delamere, and J. T. Clarke (2002), Excitation of the FUV Io tail on Jupiter: Characterization of the electron precipitation, J. Geophys. Res., 107(A11), 1394, doi:10.1029/ 2002JA009410.

Gérard, J.-C., E. J. Bunce, D. Grodent, S. W. H. Crowley, J. T. Clarke, and S. V. Badman (2005), Signature of Saturn's auroral cusp: Simultaneous Hubble Space Telescope FUV observations and upstream solar wind monitoring, J. Geophys. Res., 110, A11201, doi:10.1029/2005JA011094. Gérard, J.-C., D. Grodent, J. Gustin, A. Saglam, J. T. Clarke, and J. T. Trauger (2004), Characteristics of Saturn's FUV aurora observed with the Space Telescope Imaging Spectrograph, J. Geophys. Res., 109, A09207, doi:10.1029/2004JA010513.

Grodent, D., J.-C. Gérard, S. W. H. Cowley, E. J. Bunce, and J. T. Clarke (2005), The global morphology of Saturn's southern ultraviolet aurora, J. Geophys. Res., 110, A01207, doi:10.1029/2004JA010717.

Gurnett, D. A., et al. (2004), The Cassini Radio and Plasma Wave Investigation, Space Sci. Rev., 114, 395.

Jackman, C. M., and S. W. H. Cowley (2006), A model of the plasma flow and current in Saturn's polar ionosphere under conditions of strong Dungey-cycle driving, Ann. Geophys., 24, 1029.

Kurth, W. S., et al. (2005), An Earth-like correspondence between Saturn's auroral features and radio emission, Nature, 433, 722.

McGrath, M. A., and J. T. Clarke (1992), H I Lyman alpha emission from Saturn (1980-1990), J. Geophys. Res., 103, 20,237.

Mitchell, D. G., et al. (2005), Energetic ion acceleration in Saturn's magnetotail: Substorms at Saturn?, Geophys. Res. Lett., 32, L20S01, doi:10.1029/2005GL022647.

Sandel, B. R., and A. L. Broadfoot (1981), Morphology of Saturn's aurora, Nature, 292, 679.

Sandel, B. R., et al. (1982), Extreme ultraviolet observations from the Voyager 2 encounter with Saturn, Science, 215, 548.

Slavin, J. A., E. J. Smith, J. R. Spreiter, and S. S. Stahara (1985), Solar wind flow about the outer planets: Gas dynamic modeling of the Jupiter and Saturn bow shocks, J. Geophys. Res., 90, 6275.

Trauger, J. T., et al. (1998), Saturn's hydrogen aurora: Wide field and planetary camera 2 imaging from the Hubble Space Telescope, J. Geophys. Res., 103, 20,237.

Waite, J. H., Jr., T. E. Cravens, J. U. Kozyra, A. F. Nagy, S. K. Atreya, and R. H. Chen (1983), Electron precipitation and related aeronomy of the Jovian thermosphere and ionosphere, J. Geophys. Res., 88, 6143.

Young, D. T., et al. (2004), Cassini Plasma Spectrometer Investigation, Space Sci. Rev., 114, 1

E. J. Bunce, S. W. H. Cowley, and J. D. Nichols, Department of Physics and Astronomy, University of Leicester, University Road, Leicester LE1 7RH, UK.

J. T. Clarke, Department of Astronomy, Boston University, Boston, MA 02215, USA.

A. J. Coates, Mullard Space Science Laboratory, University College London, Holmbury St. Mary, Dorking, Surrey, RH5 6NT, UK.

F. J. Crary, Division of Space Science and Engineering, Southwest Research Institute, 9503 W. Commerce, San Antonio, TX 78227-1301, USA.

M. K. Dougherty, Imperial College London, Exhibition Road, London, SW7 2BW, UK

J.-C. Gérard and D. Grodent, Laboratoire de Physique Atmosphérique et Planétaire Université de Liège, 17 allèe du 6 août, B-4000, Liège, Belgium. (jc.gerard@ulg.ac.be)

W. S. Kurth, Department of Physics and Astronomy, University of Iowa, Iowa City, IA 52242, USA.

D. G. Mitchell, Johns Hopkins University Applied Physics Laboratory, 11100 Johns Hopkins Road, Laurel, MD 20723, USA. 